\title{
CAPM Option Pricing
}

\author{
Sven Husmann ${ }^{\mathrm{a}}$, Neda Todorova ${ }^{\mathrm{b}}$ \\ ${ }^{a}$ Department of Business Administration, European University Viadrina, \\ Große Scharrnstraße 59, D-15230 Frankfurt (Oder), Germany, \\ Email: husmann@europa-uni.de, \\ Telephone: +49(0)335 5534-2985/-2201, Fax: +49 (0)335 5534-2287. \\ ${ }^{b}$ Corresponding author. Department of Business Administration, \\ European University Viadrina, \\ Große Scharrnstraße 59, \\ D-15230 Frankfurt (Oder), Germany, \\ Email: todorova@europa-uni.de \\ Telephone: +49 (0)335 5534-2985/-2988, Fax: +49 (0)335 5534-2287. \\ We thank Nicole Apitz and Wolfgang Schmid for helpful comments.
}

\begin{abstract}
This paper extends the option pricing equations of Black and Scholes (1973), Jarrow and Madan (1997) and Husmann and Stephan (2007). In particular, we show that the length of the individual planning horizon is a determinant of an option's value. The derived pricing equations can be presented in terms of the Black and Scholes (1973) option values which ensures an easy application in practice.
\end{abstract}

Keywords: Capital Asset Pricing Model; Option Pricing; Planning Horizon; Incomplete Markets.

JEL Classification: G13 


\section{Introduction}

Options are redundant securities in a complete market. However, the empirical results of Vanden (2004) indicate that options are nonredundant in explaining returns on risky assets. In incomplete lognormal markets, Jarrow and Madan (1997) derive a CAPM option price equation. This study extends their results in three respects. First, a pricing equation is derived for an option on an arbitrary non-dividend asset, whereas the option pricing formula by Jarrow and Madan (1997) is only relevant for options on the market portfolio. Secondly, Jarrow and Madan (1997) assume that the planning horizon of the investor exceeds the time-to-maturity of the option. This study also comprises the case in which the option is sold before maturity. Husmann and Stephan (2007) consider a planning horizon equal to time-to-maturity. For a planning horizon longer or shorter than the option's time-to-maturity, we obtain two distinct formulas, making reference to the results presented by Rubinstein (1984) and Husmann and Stephan (2007). The formulas coincide solely when the planning horizon is exactly equal to the time-to-maturity of the option or when the market is complete. Thirdly, the value of an European option for all considered cases can be presented in terms of the Black and Scholes (1973) values of a number of options. This ensures that the results are easy to use in practice.

Sections 2 presents the notation and assumptions used. Section 3 indicates the theoretical results, and Section 4 concludes.

\section{Assumptions and Notation}

The valuation of options in section 3 is based on the default assumptions of the CAPM. Additionally, we assume that the instantaneous rate of return on any asset and the market portfolio have a joint normal distribution.

The following notation is used throughout the article:

$h \quad$ Investor's planning horizon

$\tau$ Time-to-maturity of an option

$K \quad$ Exercise price on an option

$S_{0} \quad$ Price of an underlying asset $\mathrm{S}$

$C_{0} \quad$ Price of a call on an asset $\mathrm{S}$

$\tilde{S}_{t} \quad$ Cash flow of the underlying asset

$\tilde{C}_{t} \quad$ Cash flow of the call

$\tilde{R}_{m} \quad$ Standardized cash flow of the market portfolio 
$r \quad$ Instantaneous risk-free rate of interest

$\tilde{r}_{s} \quad$ Instantaneous rate of return on asset $\mathrm{S}$

$\tilde{r}_{m} \quad$ Instantaneous rate of return on the market index

$\mu_{s} \quad$ Expected instantaneous rate of return on asset $\mathrm{S}$

$\mu_{m} \quad$ Expected instantaneous rate of return on the market index

$\sigma_{s} \quad$ Instantaneous variance of the rate of return on asset $\mathrm{S}$

$\sigma_{m} \quad$ Instantaneous variance of the rate of return on the market index

Consider the case of a call option on an arbitrary non-dividend asset S maturing at time $s$ when the planning horizon of the individual investor extends to time $t$. Our aim is to evaluate this option at time 0 . Therefore, the timeto-maturity of the option equals $\tau=s-0$ and the length of the planning horizon is $h=t-0$.

Under the given parameters for a bivariate normal distribution of rates of return, the following expressions represent the expected values, variances and covariances of the securities' cash flow and market portfolio's standardized cash flow over a period with length $h^{1}$

$$
\begin{aligned}
E\left[\tilde{S}_{t}\right] & =S_{0} e^{\left(\mu_{m}+\frac{1}{2} \sigma_{s}^{2}\right) h}, \\
E\left[\tilde{R}_{m}\right] & =e^{\left(\mu_{m}+\frac{1}{2} \sigma_{m}^{2}\right) h} \\
\operatorname{Var}\left[\tilde{R}_{m}\right] & =e^{\left(2 \mu_{m}+\sigma_{m}^{2}\right) h}\left(e^{\sigma_{m}^{2} h}-1\right), \\
\operatorname{Cov}\left[\tilde{S}_{t}, \tilde{R}_{m}\right] & =S_{0} e^{\left(\mu_{m}+\frac{1}{2} \sigma_{m}^{2}+\mu_{s}+\frac{1}{2} \sigma_{s}^{2}\right) h}\left(e^{\rho \sigma_{m} \sigma_{s} h}-1\right) .
\end{aligned}
$$

It is assumed that the parameters of the price process remain constant during the planning horizon. Furthermore, for the sake of clarity, we use the following notation for the Black and Scholes (1973) price of a call with time-tomaturity $y$ when the price of the current asset is replaced by $S_{0} e^{\left(\mu_{s}+\frac{1}{2} \sigma_{s}^{2}\right) y+x}$, the strike price is replaced by $K e^{r y}$ and the volatility of the asset amounts to $\sigma_{s}$ :

$$
\mathrm{C}[x, y]=C_{0}^{B S}\left(S_{0} e^{\left(\mu_{s}+\frac{1}{2} \sigma_{s}^{2}\right) y+x}, K e^{r y}, y, r, \sigma_{s}\right)
$$

\footnotetext{
${ }^{1}$ To calculate the moments of the lognormal distribution, see Appendix A in Husmann and Stephan (2007).
} 


\section{CAPM Option Pricing}

In an incomplete lognormal market, the CAPM may be used for option pricing. The well-known certainty equivalent valuation formula of the CAPM is

$$
\mathrm{C}_{0}=\frac{E\left[\tilde{C}_{t}\right]-\lambda \cdot \operatorname{Cov}\left[\tilde{C}_{t}, \tilde{R}_{m}\right]}{e^{r \cdot h}} \quad \text { mit } \quad \lambda_{h}=\frac{E\left[\tilde{R}_{m}\right]-e^{r \cdot h}}{\operatorname{Var}\left[\tilde{R}_{m}\right]}
$$

To be able to use this equation to value a call on $\mathrm{S}$, we must first determine the expected cash flow of the call and the covariance between the cash flow of the call and the rate of return for the market portfolio. The expected cash flow of the call is

$$
\begin{aligned}
E\left[\tilde{C}_{t}\right] & =\int_{-\infty}^{\infty} \tilde{C}_{t} f\left(r_{s}\right) d r_{s}, \\
\text { where } \quad f\left(r_{s}\right) & =\frac{1}{\sigma_{s} \sqrt{h} \sqrt{2 \pi}} e^{-\frac{1}{2}\left(\frac{r_{s}-\mu_{s} h}{\sigma_{s} \sqrt{h}}\right)^{2}} .
\end{aligned}
$$

We can simplify the calculation of the covariance using the decomposition theorem,

$$
\operatorname{Cov}\left[\tilde{C}_{t}, \tilde{R}_{m}\right]=E\left[\tilde{C}_{t} \cdot \tilde{R}_{m}\right]-E\left[\tilde{C}_{t}\right] \cdot E\left[\tilde{R}_{m}\right]
$$

$E\left[\tilde{R}_{m}\right]$ and $E\left[\tilde{C}_{t}\right]$ are to be obtained from (2) and (7) and

$$
E\left[\tilde{C}_{t} \cdot \tilde{R}_{m}\right]=\int_{-\infty}^{\infty} \int_{-\infty}^{\infty} \tilde{C}_{t} \cdot e^{r_{m}} f\left(r_{s}, r_{m}\right) d r_{m} d r_{s}
$$

where the joint density function of the bivariate normal distribution $f\left(r_{s}, r_{m}\right)$ equals

$$
\frac{1}{2 \pi \sqrt{\sigma_{s}^{2} \sigma_{m}^{2} h^{2}\left(1-\rho^{2}\right)}} \cdot e^{-\frac{1}{2\left(1-\rho^{2}\right)}\left(\frac{\left(r_{s}-\mu_{s} h\right)^{2}}{\sigma_{s}^{2} h}-2 \rho \frac{\left(r_{s}-\mu_{s} h\right)\left(r_{m}-\mu_{m} h\right)}{\sigma_{s} \sigma_{m} h}+\frac{\left(r_{m}-\mu_{m} h\right)^{2}}{\sigma_{m}^{2} h}\right)} .
$$

Furthermore, (2) and (3) give the following for the market price of risk

$$
\lambda_{h}=\frac{e^{\left(\mu_{m}+\frac{1}{2} \sigma_{m}^{2}\right) h}-e^{r h}}{e^{\left(2 \mu_{m}+\sigma_{m}^{2}\right) h}\left(e^{\sigma_{m}^{2} h}-1\right)} .
$$


In the following, we discern three cases. First, it is assumed that the investor's planning horizon is longer than to the time-to-maturity of the option $(h>\tau)$. Then, the investor's planning horizon coincides with the time-to-maturity of the option $(h=\tau)$. Last, we assume that the option is sold before maturity $(h<\tau)$ so that the remaining time-to-maturity is $(\tau-h)$. We establish pricing equations for call options. Put prices can be derived in an analogue way.

\subsection{Planning horizon longer than the option's time-to-maturity}

\subsubsection{Expected value}

Because any contingent claim paying $\max \left(S_{0} e^{r_{s}}-K, 0\right)$ at time $s$ is equivalent to one paying $\max \left(S_{0} e^{r_{s}}-K, 0\right) e^{r(h-\tau)}$ at time $t$, the expected value of the call amounts to ${ }^{2}$

$$
\begin{aligned}
E\left[\tilde{C}_{t} \mid \mathcal{F}_{0}\right] & =\int_{-\infty}^{\infty} \max \left(S_{0} e^{r_{s}}-K, 0\right) e^{r(h-\tau)} f\left(r_{s}\right) d r_{s} \\
& =e^{r(h-\tau)}\left(S_{0} e^{\left(\mu_{s}+\frac{1}{2} \sigma_{s}^{2}\right) \tau} N\left(d_{1}\right)-K N\left(d_{2}\right)\right), \\
\text { where } \quad d_{1} & =\frac{\ln \left(\left(S_{0} e^{\left(\mu_{s}+\frac{1}{2} \sigma_{s}^{2}\right) \tau}\right) /\left(K e^{r \tau}\right)\right)+\left(r+\frac{1}{2} \sigma_{s}^{2}\right) \tau}{\sigma_{s} \sqrt{\tau}}, \\
d_{2} & =d_{1}-\sigma_{s} \sqrt{\tau} .
\end{aligned}
$$

Therefore, the expected cash flow of a call can be presented as a special case of the compounded option valuation equation of Black and Scholes (1973), except that it is evaluated at

$$
\begin{aligned}
E\left[\tilde{C}_{t} \mid \mathcal{F}_{0}\right] & =e^{r(h-\tau)} C_{0}^{B S}\left(S_{0} e^{\left(\mu_{s}+\frac{1}{2} \sigma_{s}^{2}\right) \tau}, K e^{r \tau}, \tau, r, \sigma_{s}\right) \\
& =e^{r(h-\tau)} \mathrm{C}[0, \tau]
\end{aligned}
$$

\subsubsection{Covariance}

To establish the covariance between the cash flow of the call and the rate of return on the market portfolio using the decomposition theorem, we first

\footnotetext{
${ }^{2}$ For a detailed deviation in the case $h=\tau$, see appendix B in Husmann and Stephan (2007). The results can be easily extended to hold for $h>\tau$. To present the results of Husmann and Stephan (2007) in terms of Black and Scholes (1973) prices, some conversions are necessary.
} 
compute $E\left[\tilde{C}_{t} \cdot \tilde{R}_{m} \mid \mathcal{F}_{0}\right]$. Our task is to integrate

$$
\int_{-\infty}^{\infty} \int_{-\infty}^{\infty} \max \left(S_{0} e^{r_{s}}-K, 0\right) e^{r(h-\tau)} e^{r_{m}} e^{\left(\mu_{m}+\frac{1}{2} \sigma_{m}^{2}\right)(h-\tau)} f\left(r_{s}, r_{m}\right) d r_{m} d r_{s}
$$

Integration yields (see Appendix B in Husmann and Stephan (2007), with minor conversions) the following expression for $E\left[\tilde{C}_{t} \cdot \tilde{R}_{m} \mid \mathcal{F}_{0}\right]$ :

$$
\begin{aligned}
e^{\left(\mu_{m}+\frac{1}{2} \sigma_{m}^{2}\right) h+r(h-\tau)} & \left(S_{0} e^{\left(\mu_{s}+\frac{1}{2} \sigma_{s}^{2}+\rho \sigma_{s} \sigma_{m}\right) \tau} N\left(d_{3}\right)-K N\left(d_{4}\right)\right) \\
\text { where } \quad d_{3} & =\frac{\ln \left(\left(S_{0} e^{\left(\mu_{s}+\frac{1}{2} \sigma_{s}^{2}+\rho \sigma_{s} \sigma_{m}\right) \tau}\right) /\left(K e^{r \tau}\right)\right)+\left(r+\frac{1}{2} \sigma_{s}^{2}\right) \tau}{\sigma_{s} \sqrt{\tau}} \\
d_{4} & =d_{2}-\sigma_{s} \sqrt{\tau} .
\end{aligned}
$$

It follows that

$$
E\left[\tilde{C}_{t} \cdot \tilde{R}_{m} \mid \mathcal{F}_{0}\right]=e^{\left(\mu_{m}+\frac{1}{2} \sigma_{m}^{2}\right) h+r(h-\tau)} \mathrm{C}\left[\rho \sigma_{m} \sigma_{s} \tau, \tau\right]
$$

and accordingly,

$$
\operatorname{Cov}\left[\tilde{C}_{t}, \tilde{R}_{m} \mid \mathcal{F}_{0}\right]=e^{\left(\mu_{m}+\frac{1}{2} \sigma_{m}^{2}\right) h+r(h-\tau)}\left(\mathrm{C}\left[\rho \sigma_{s} \sigma_{m} \tau, \tau\right]-\mathrm{C}[0, \tau]\right) .
$$

\subsubsection{Valuation equation}

With (11) and (16) employed in (6), the CAPM option price equation is

$$
C_{0}=\frac{\mathrm{C}[0, \tau]-\lambda_{h} e^{\left(\mu_{m}+\frac{1}{2} \sigma_{m}^{2}\right) h}\left(\mathrm{C}\left[\rho \sigma_{m} \sigma_{s} \tau, \tau\right]-\mathrm{C}[0, \tau]\right)}{e^{r \cdot \tau}} .
$$

\subsubsection{Special cases}

For calls on the market portfolio, the following applies, $\rho=1, \sigma_{m}=\sigma_{s}$ and $\mu_{m}=\mu_{s}$. The valuation equation shown in (17) can be easily transformed into

$$
C_{0}=\frac{\mathrm{C}[0, \tau]-\lambda_{h} e^{\left(\mu_{m}+\frac{1}{2} \sigma_{m}^{2}\right) h}\left(\mathrm{C}\left[\sigma_{m}^{2} \tau, \tau\right]-\mathrm{C}[0, \tau]\right)}{e^{r \tau}} .
$$

After some conversions, we see that (18) and the valuation equation of Jarrow and Madan $(1997)^{3}$ are identical. However, Jarrow and Madan (1997) define

\footnotetext{
${ }^{3}$ Equation (20), p. 21.
} 
the parameter $\mu$ as the rate of return of the expected value, whereas we use it to identify the expected rate of return. To make our results comparable with theirs, the parameter $\mu$ must be replaced with $\mu+\sigma^{2} / 2$ in Jarrow and Madan (1997).

In complete markets, a risk-neutral valuation always leads to the correct valuation results (see Cox and Ross 1976). As in risk-neutral settings $\mu_{m}+$ $\frac{1}{2} \sigma_{m}^{2}=\mu_{s}+\frac{1}{2} \sigma_{s}^{2}=r$, one can use $\lambda_{h}=0$ to obtain the Black and Scholes (1973) price of the option:

$$
C_{0}=\frac{C_{0}^{B S}\left(S_{0} e^{\left(\mu_{s}+\frac{1}{2} \sigma_{s}^{2}\right) \tau}, K e^{r \tau}, \tau, r, \sigma_{s}\right)}{e^{r \tau}}=C_{0}^{B S}\left(S_{0}, K, \tau, r, \sigma_{s}\right) .
$$

\subsection{Planning horizon equal to the option's time-to-maturity}

When the option is held until maturity $(h=\tau)$, the valuation equation (17) can be simplified to

$$
\begin{gathered}
C_{0}=\frac{\mathrm{C}[0, \tau]-\lambda_{\tau} e^{\left(\mu_{m}+\frac{1}{2} \sigma_{m}^{2}\right) \tau}\left(\mathrm{C}\left[\rho \sigma_{s} \sigma_{m} \tau, \tau\right]-\mathrm{C}[0, \tau]\right)}{e^{r \tau}}, \\
\text { where } \quad \lambda_{\tau}=\frac{e^{\left(\mu_{m}+\frac{1}{2} \sigma_{m}^{2}\right) \tau}-e^{r \tau}}{e^{\left(2 \mu_{m}+\sigma_{m}^{2}\right) \tau}\left(e^{\sigma_{m}^{2} \tau}-1\right)}
\end{gathered}
$$

This pricing formula coincides with the one derived by Husmann and Stephan (2007), except that the latter is not presented in terms of Black and Scholes (1973) prices.

\subsection{Planning horizon shorter than the option's time-to-maturity}

When the planning horizon is shorter that the time-to-maturity, we use the results presented by Rubinstein (1984) who obtained the expected value of the call for $\rho=0$ in terms of a Black and Scholes (1973) value of an European-style call as ${ }^{4}$

$$
E\left[\tilde{C}_{t} \mid \mathcal{F}_{0}\right]=C_{0}^{B S}\left(S_{0} e^{\left(\mu_{s}+\frac{1}{2} \sigma_{s}^{2}\right) h}, K e^{r h}, \tau, r, \sigma_{s}\right)
$$

\footnotetext{
${ }^{4}$ The deviation is based on a minor extension of a known integral of the normal distribution, see Owen (1981), p. 403, integral 10,010.8. The proof is available from the authors on request.
} 


\subsubsection{Expected value}

We can express the expected value of a call at the end of the holding period using the results obtained using the equation in (20). Our task is to integrate

$$
\int_{-\infty}^{\infty} \frac{C_{0}^{*}-\lambda_{\tau-h} e^{\left(\mu_{m}+\frac{1}{2} \sigma_{m}^{2}\right)(\tau-h)}\left(C_{0}^{* *}-C_{0}^{*}\right)}{e^{r(\tau-h)}} f\left(r_{s}\right) d r_{s}
$$

where $C_{0}^{*}=\mathrm{C}\left[\tilde{r}_{s}, \tau-h\right]$,

$$
C_{0}^{* *}=\mathrm{C}\left[\tilde{r}_{s}+\rho \sigma_{s} \sigma_{m}(\tau-h), \tau-h\right],
$$

$$
\lambda_{\tau-h}=\frac{e^{\left(\mu_{m}+\frac{1}{2} \sigma_{m}^{2}\right)(\tau-h)}-e^{r(\tau-h)}}{e^{\left(2 \mu_{m}+\sigma_{m}^{2}\right)(\tau-h)}\left(e^{\sigma_{m}^{2}(\tau-h)}-1\right)} .
$$

Splitting (26) in three integrals and utilizing the solution presented by $R u$ binstein (1984) gives us

$$
\begin{aligned}
E\left[\tilde{C}_{t} \mid \mathcal{F}_{0}\right] & =\frac{C_{0}^{1}-\lambda_{\tau-h} e^{\left(\mu_{m}+\frac{1}{2} \sigma_{m}^{2}\right)(\tau-h)}\left(C_{0}^{2}-C_{0}^{1}\right)}{e^{r(\tau-h)}}, \\
\text { where } \quad C_{0}^{1} & =\mathrm{C}[0, \tau], \\
C_{0}^{2} & =\mathrm{C}\left[\rho \sigma_{s} \sigma_{m}(\tau-h), \tau\right] .
\end{aligned}
$$

\subsubsection{Covariance}

Again, we compute the covariance between the value of the call and the return on the market portfolio at the end of the holding period using the decomposition theorem. First, to compute $E\left[\tilde{C}_{t} \cdot \tilde{R}_{m} \mid \mathcal{F}_{0}\right]$, we must integrate

$$
\int_{-\infty}^{\infty} \int_{-\infty}^{\infty} \frac{C_{0}^{*}-\lambda_{\tau-h} e^{\left(\mu_{m}+\frac{1}{2} \sigma_{m}^{2}\right)(\tau-h)}\left(C_{0}^{* *}-C_{0}^{*}\right)}{e^{r(\tau-h)}} e^{r_{m}} f\left(r_{s}, r_{m}\right) d r_{s} d r_{m}
$$

Integration yields the following: ${ }^{5}$

$$
\begin{aligned}
E\left[\tilde{C}_{t} \cdot \tilde{R}_{m} \mid \mathcal{F}_{0}\right] & =e^{\left(\mu_{m}+\frac{1}{2} \sigma_{m}^{2}\right) h} \frac{C_{0}^{3}-\lambda_{\tau-h} e^{\left(\mu_{m}+\frac{1}{2} \sigma_{m}^{2}\right)(\tau-h)}\left(C_{0}^{4}-C_{0}^{3}\right)}{e^{r(\tau-h)}}, \\
\text { where } \quad C_{0}^{3} & =\mathrm{C}\left[\rho \sigma_{m} \sigma_{s} h, \tau\right] \\
C_{0}^{4} & =\mathrm{C}\left[\rho \sigma_{m} \sigma_{s} \tau, \tau\right] .
\end{aligned}
$$

\footnotetext{
${ }^{5}$ The proof is available from the authors on request.
} 
The covariance $\operatorname{Cov}\left[\tilde{C}_{t}, \tilde{R}_{m} \mid \mathcal{F}_{0}\right]$ is thus given by

$$
e^{\left(\mu_{m}+\frac{1}{2} \sigma_{m}^{2}\right) h} \cdot \frac{\left(C_{0}^{3}-C_{0}^{1} \lambda_{\tau-h} e^{\left(\mu_{m}+\frac{1}{2} \sigma_{m}^{2}\right)(\tau-h)}\left(C_{0}^{4}-C_{0}^{2}-C_{0}^{3}+C_{0}^{1}\right)\right)}{e^{r(\tau-h)}} .
$$

\subsubsection{Valuation equation}

Using (27) and (34) in

$$
C_{0}=e^{-r h}\left(E\left[\tilde{C}_{t} \mid \mathcal{F}_{0}\right]-\lambda_{h} \operatorname{Cov}\left[\tilde{C}_{t}, \tilde{R}_{m} \mid \mathcal{F}_{0}\right]\right)
$$

with

$$
\lambda_{h}=\frac{e^{\left(\mu_{m}+\frac{1}{2} \sigma_{m}^{2}\right) h}-e^{r h}}{e^{\left(2 \mu_{m}+\sigma_{m}^{2}\right) h}\left(e^{\sigma_{m}^{2} h}-1\right)}
$$

results in

$$
\begin{aligned}
C_{0}= & e^{-r \tau}\left(C_{0}^{1}-\lambda_{\tau-h} e^{\left(\mu_{m}+\frac{1}{2} \sigma_{m}^{2}\right)(\tau-h)}\left(C_{0}^{2}-C_{0}^{1}\right)\right. \\
& -\lambda_{h} e^{\left(\mu_{m}+\frac{1}{2} \sigma_{m}^{2}\right) h}\left(C_{0}^{3}-C_{0}^{1}\right) \\
& \left.+\lambda_{\tau-h} \lambda_{h} e^{\left(\mu_{m}+\frac{1}{2} \sigma_{m}^{2}\right) \tau}\left(C_{0}^{1}+C_{0}^{4}-C_{0}^{2}-C_{0}^{3}\right)\right),
\end{aligned}
$$

with $C_{0}^{1}-C_{0}^{4}$ given in (28), (29), (32) and (33) and $\lambda_{\tau-h}$ in (26).

\subsubsection{Special cases}

When the holding period is equal to the time-to-maturity of the option $(h=\tau)$, it follows that $C_{0}^{1}=C_{0}^{2}$ und $C_{0}^{3}=C_{0}^{4}$. Therefore, (37) becomes

$$
C_{0}=\frac{C_{0}^{1}-\lambda_{h} e^{\left(\mu_{m}+\frac{1}{2} \sigma_{m}^{2}\right) \tau}\left(C_{0}^{3}-C_{0}^{1}\right)}{e^{r \tau}},
$$

and is hence identical to (20).

In a risk-neutral world, because $\mu_{m}+\frac{1}{2} \sigma_{m}^{2}=\mu_{s}+\frac{1}{2} \sigma_{s}^{2}=r$, one can again obtain the Black and Scholes (1973) price using $\lambda_{h}=\lambda_{\tau-h}=0$,

$$
C_{0}=\frac{C_{0}^{B S}\left(S_{0} e^{\left(\mu_{s}+\frac{1}{2} \sigma_{s}^{2}\right) \tau}, K e^{r \tau}, \tau, r, \sigma_{s}\right)}{e^{r \tau}}=C_{0}^{B S}\left(S_{0}, K, \tau, r, \sigma_{s}\right) .
$$




\section{Conclusion}

This paper extends the valuation results presented by Jarrow and Madan (1997), considering the general case of an option on an arbitrary asset in an incomplete lognormal market. Additionally, we consider the case in which the planning horizon of the individual investor is shorter that the time-tomaturity of the option. The case in which the planning horizon equals the option's time-to-maturity is included in Husmann and Stephan (2007). The derived pricing equations depend explicitly on the planning horizon of the individual investor and are easy to use due to their simple presentation. In complete markets, the pricing equations for an European call result in the well-known Black and Scholes (1973) price.

Black, F., Scholes, M., 1973. The Pricing of Options and Corporate Liabilities. Journal of Political Economy 81, 637-654.

Cox, J., Ross, S., 1976. The Valuation of Options for Alternative Stochastic Processes. Journal of Financial Economics 3, 145-166.

Husmann, S., Stephan, A., 2007. On Estimating an Asset's Implicit Beta. Journal of Futures Markets 27, 961-979.

Jarrow, R. A., Madan, D. B., 1997. Is Mean-Variance Analysis Vacuous: Or was Beta Still Born? European Finance Review 1, 15-30.

Owen, D. B., 1981. A table of normal integrals. Communications in Statistics - Simulation and Computation 9, 389-419.

Rubinstein, M., 1984. A Simple Formula for the Expected Rate of Return of an Option over a Finite Holding Period. Journal of Finance 39, 1503-1509.

Vanden, J. M., 2004. Options Trading and the CAPM. Review of Financial Studies 17, 207-239. 